\title{
A CONSTRUCTIVE METHOD TO CONTROLLABILITY AND OBSERVABILITY FOR QUASILINEAR HYPERBOLIC SYSTEMS*
}

\author{
TATSIEN LI (DAQIAN LI) ${ }^{\dagger}$
}

\begin{abstract}
A simple constructive method is presented to get the exact boundary controllability, the exact boundary controllability of nodal profile and the exact boundary observability for 1-D quasilinear hyperbolic systems.
\end{abstract}

Key words. Exact boundary controllability, Exact boundary controllability of nodal profile, Exact boundary observability, Quasilinear hyperbolic system, Contructive method.

AMS subject classifications. 35B37, 93B05, 93B07, 35L50

1. Introduction and preliminaries. The controllability and observability are of great importance in both theory and applications. A complete theory has been established for linear hyperbolic systems, in particular, for linear wave equations [2527]. There have also been some results for semilinear wave equations [4, 11, 29-30]. For quasilinear hyperbolic systems that have numerous applications in mechanics, physics and other applied sciences, however, very few results are available even in one-space-dimensional case $[2-3]$.

In this paper we will present a simple and efficient constructive method to the exact boundary controllability, the exact boundary controllability of nodal profile and the exact boundary observability for general 1-D quasilinear hyperbolic systems with general nonlinear boundary conditions [12, 14-17, 19-21].

Noting that for the weak solution to quasilinear hyperbolic systems, which includes shock waves and corresponds to an irreversible process, generically speaking, it is impossible to have the exact boundary controllability for any arbitrarily given initial and final states [1]. In order to give a general and systematic presentation, we consider only the classical solution to quasilinear hyperbolic systems, which corresponds to a reversible process.

Since this method is given in the framework of classical solutions, only the local exact boundary controllability and the local exact boundary observability can be obtained generically, however, in the special case that the problem is linear, this method will directly lead to the global exact boundary controllability and the global exact boundary observability.

In what follows, we consider only the case of first order quasilinear hyperbolic systems, for higher order quasilinear hyperbolic systems the corresponding problem can be discussed in a similar way [13, 22-23, 28].

Consider the following 1-D first order quasilinear hyperbolic system

$$
\frac{\partial u}{\partial t}+A(u) \frac{\partial u}{\partial x}=B(u)
$$

where $u=\left(u_{1}, \ldots, u_{n}\right)^{T}$ is the unknown vector function of $(t, x), A(u)$ is a given $n \times n$ matrix with $C^{1}$ elements $a_{i j}(u)(i, j=1, \ldots, n)$ and $B(u)=\left(b_{1}(u), \ldots, b_{n}(u)\right)^{T}$ is a given $C^{1}$ vector function of $u$.

${ }^{*}$ Received September 2, 2010; accepted for publication January 20, 2011. Supported by the Basic Research Program of China (No. 2007CB814800).

${ }^{\dagger}$ School of Mathematical Sciences, Fudan University, Shanghai 200433, China; Shanghai Key Laboratory for Contemporary Applied Mathematics; Nonlinear Mathematical Modeling and Methods Laboratory (dqli@fudan.edu.cn). 
By hyperbolicity, for any given $u$ on the domain under consideration, $A(u)$ possesses $n$ real eigenvalues $\lambda_{1}(u), \ldots, \lambda_{n}(u)$ and a complete set of left eigenvectors $l_{i}(u)=\left(l_{i 1}(u), \ldots, l_{i n}(u)\right)(i=1, \ldots, n)$ :

$$
l_{i}(u) A(u)=\lambda_{i}(u) l_{i}(u)
$$

We suppose that all $\lambda_{i}(u)$ and $l_{i}(u)(i=1, \ldots, n)$ have the same $C^{1}$ regularity as $A(u)=\left(a_{i j}(u)\right)$.

Suppose that on the domain under consideration there are no zero eigenvalues:

$$
\lambda_{r}(u)<0<\lambda_{s}(u) \quad(r=1, \ldots, m ; s=m+1, \ldots, n),
$$

and, for simplicity of statement, we assume that the number of positive eigenvalues is equal to that of negative ones:

$$
n-m=m, \quad \text { i.e., } \quad n=2 m \text {. }
$$

We now give all the basic ingredients of our constructive method as follows.

\section{A. Semi-global $C^{1}$ solution to the mixed initial-boundary value prob-} lem $[16,18]$.

Let

$$
v_{i}=l_{i}(u) u \quad(i=1, \ldots, n) .
$$

$v_{i}$ is called to be the diagonalized variable corresponding to the $i$-th characteristic

$$
\frac{\mathrm{d} x}{\mathrm{~d} t}=\lambda_{i}(u)
$$

On the domain $\{(t, x) \mid t \geq 0,0 \leq x \leq L\}$ we consider the forward mixed initialboundary value problem for system (1.1) with the initial condition

$$
t=0: u=\varphi(x), \quad 0 \leq x \leq L
$$

and the following boundary conditions

$$
\begin{array}{ll}
x=0: & v_{s}=G_{s}\left(t, v_{1}, \ldots, v_{m}\right)+H_{s}(t) \quad(s=m+1, \ldots, n), \\
x=L: & v_{r}=G_{r}\left(t, v_{m+1}, \ldots, v_{n}\right)+H_{r}(t) \quad(r=1, \ldots, m),
\end{array}
$$

where $\varphi, G_{i}$ and $H_{i}(i=1, \ldots, n)$ are all $C^{1}$ functions with respect to their arguments and, without loss of generality, we assume

$$
G_{i}(t, 0, \ldots, 0) \equiv 0 \quad(i=1, \ldots, n) .
$$

We point out that (1.7) and (1.8) are the most general nonlinear boundary conditions to guarantee the well-posedness for the forward mixed problem, the characters of which can be shown as

1 ) The number of boundary conditions on $x=0$ (resp. on $x=L$ ) is equal to the number of positive (resp. negative) eigenvalues.

These characteristics which reach the corresponding boundary from the interior of the domain are called to be the coming characteristics, while, all other characteristics which enter the domain from the corresponding boundary are called to be the departing characteristics. 
Thus, the first character can be restated as

1) The number of boundary conditions on $x=0$ (resp. on $x=L$ ) is equal to the number of coming characteristics on it.

2) The boundary conditions on $x=0$ (resp. on $x=L$ ) are written in the form that all the diagonalized variables corresponding to the coming characteristics are explicitly expressed by other diagonalized variables corresponding to the departing characteristics.

When the conditions of $C^{1}$ compatibility are satisfied at the point $(t, x)=(0,0)$ and $(0, L)$, respectively, the froward mixed problem (1.1) and (1.6)-(1.8) admits a unique local $C^{1}$ solution $u=u(t, x)$ on the domain $R\left(\delta_{0}\right)=\left\{(t, x) \mid 0 \leq t \leq \delta_{0}, 0 \leq\right.$ $x \leq L\}$, where $\delta_{0}>0$ is a suitably small number [24]. However, in order to realize the exact boundary controllability and the exact boundary observability, we need the $C^{1}$ solution $u=u(t, x)$ to the mixed problem on a domain $R\left(T_{0}\right)=\{(t, x) \mid 0 \leq t \leq$ $\left.T_{0}, 0 \leq x \leq L\right\}$, where $T_{0}>0$ is a preassigned and possibly quite large number. This kind of $C^{1}$ solution, which is neither a local $C^{1}$ solution nor a global $C^{1}$ solution, is called to be a semi-global $C^{1}$ solution. In order to guarantee the existence of semiglobal $C^{1}$ solution, some additional hypotheses are needed. We have the following

LEMMA 1.1 (Semi-global $C^{1}$ solution to the forward mixed problem). Under the previous assumptions, suppose furthermore that

$$
B(0)=0,
$$

namely, $u=0$ is an equilibrium of system (1.1). For any given $T_{0}>0$, if the $C^{1}$ norms $\|\varphi\|_{C^{1}[0, L]}$ and $\|H\|_{C^{1}\left[0, T_{0}\right]}$ (in which $\left.H(t)=\left(H_{1}(t), \ldots, H_{n}(t)\right)^{T}\right)$ are small enough (depending on $T_{0}$ ), the forward mixed initial-boundary value problem (1.1) and (1.6)-(1.8) admits a unique semi-global $C^{1}$ solution $u=u(t, x)$ with small $C^{1}$ norm on the domain $R\left(T_{0}\right)=\left\{(t, x) \mid 0 \leq t \leq T_{0}, 0 \leq x \leq L\right\}$. Moreover, under the additional hypotheses that $\frac{\partial G_{i}}{\partial t}(i=1, \ldots, n)$ satisfy locally the Lipschitz condition with respect to the variable $v=\left(v_{1}, \ldots, v_{n}\right)^{T}$, we have

$$
\|u\|_{C^{1}\left[R\left(T_{0}\right)\right]} \leq C\left(\|\varphi\|_{C^{1}[0, L]}+\|H\|_{C^{1}\left[0, T_{0}\right]}\right),
$$

where $C$ is a positive constant possibly depending on $T_{0}$.

For the backward mixed initial-boundary value problem for system (1.1) with the final condition

$$
t=T_{0}: \quad u=\Phi(x), \quad 0 \leq x \leq L,
$$

similar results can be obtained. However, since in this situation the original coming characteristics on the boundaries $x=0$ and $x=L$ become the departing characteristics and vice versa, we should assume that the boundary conditions (1.7)-(1.8) can be equivalently rewritten as

$$
\begin{gathered}
x=0: v_{r}=\bar{G}_{r}\left(t, v_{m+1}, \ldots, v_{n}\right)+\bar{H}_{r}(t) \quad(r=1, \ldots, m), \\
x=L: v_{s}=\bar{G}_{s}\left(t, v_{1}, \ldots, v_{m}\right)+\bar{H}_{s}(t) \quad(s=m+1, \ldots, n)
\end{gathered}
$$

with

$$
\bar{G}_{i}(t, 0, \ldots, 0) \equiv 0 \quad(i=1, \ldots, n) .
$$

B. Global $C^{1}$ solution to the Cauchy problem on a finite initial interval. 
Similarly to Lemma 1.1, we have

LEMMA 1.2 (Global $C^{1}$ solution to the Cauchy problem on a finite initial interval). Under assumption (1.10), if $\|\varphi\|_{C^{1}[0, L]}$ is small enough, then the Cauchy problem (1.1) and (1.6) admits a unique global $C^{1}$ solution $u=u(t, x)$ on the whole maximum determinate domain $D=\{(t, x) \mid t \geq 0, \bar{x}(t) \leq x \leq \overline{\bar{x}}(t)\}$ and

$$
\|u\|_{C^{1}(D)} \leq C\|\varphi\|_{C^{1}[0, L]},
$$

where $C$ is a positive constant, $x=\bar{x}(t)$ is the rightmost characteristic passing through the point $(t, x)=(0,0)$ :

$$
\left\{\begin{array}{l}
\frac{\mathrm{d} \bar{x}(t)}{\mathrm{d} t}=\max _{i=1, \ldots, n} \lambda_{i}(u(t, \bar{x}(t))) \\
\bar{x}(0)=0
\end{array}\right.
$$

and $x=\overline{\bar{x}}(t)$ is the leftmost characteristic passing through the point $(t, x)=(0, L)$ :

$$
\left\{\begin{array}{l}
\frac{\mathrm{d} \overline{\bar{x}}(t)}{\mathrm{d} t}=\min _{i=1, \ldots, n} \lambda_{i}(u(t, \overline{\bar{x}}(t))), \\
\overline{\bar{x}}(0)=L
\end{array}\right.
$$

Similar results can be obtained for the backward Cauchy problem.

\section{Uniqueness of $C^{1}$ solution to the one-sided mixed initial-boundary value problem [23].}

LEMma 1.3 (Uniqueness of $C^{1}$ solution to the one-sided mixed problem). For the one-sided mixed initial-boundary value problem (1.1) and (1.6)-(1.7), its $C^{1}$ solution $u=u(t, x)$ is unique on the maximum determinate domain $\{(t, x) \mid t \geq 0,0 \leq$ $x \leq \overline{\bar{x}}(t)\}$, where $x=\overline{\bar{x}}(t)$ is the leftmost characteristic passing through the point $(t, x)=(0, L)$, defined by $(1.18)$.

For the one-sided mixed problem (1.1), (1.6) and (1.8), similar results hold.

\section{Change the role of $t$ and $x$.}

Since there are no zero eigenvalues (see (1.3)), we can change the role of $t$ and $x$, and the original system (1.1) is rewritten as

$$
\frac{\partial u}{\partial x}+A^{-1}(u) \frac{\partial u}{\partial t}=\widetilde{B}(u) \stackrel{\text { def. }}{=} A^{-1}(u) B(u) .
$$

Thus, the matrix $A(u)$ is replaced by its inverse $A^{-1}(u)$, correspondingly, the eigenvalues $\lambda_{i}(u)(i=1, \cdots, n)$ become $\frac{1}{\lambda_{i}(u)}(i=1, \cdots, n)$, however, the left eigenvectors $l_{i}(u)(i=1, \cdots, n)$ keep unchanged, then the variables $v_{i}(i=1, \cdots, n)$ are still given by the same formula (1.5).

In this situation we have similar results for the leftward (resp. rightward) mixed problem and for the leftward (resp. rightward) Cauchy problem.

In what follows we will see that by means of the previous four basic facts, a constructive method can be flexibly used to get the desired conclusion on the exact boundary controllability, on the exact boundary controllability of nodal profile and on the exact boundary observability. 
2. Exact boundary controllability. For any given $C^{1}$ final state at $t=T$ :

$$
t=T: \quad u=\Phi(x), \quad 0 \leq x \leq L,
$$

we hope to choose suitable boundary controls $H_{i}(t)(i=1, \ldots, n)$ or a part of $H_{i}(t)(i=1, \ldots, n)$, such that the corresponding mixed initial-boundary value problem (1.1) and (1.6)-(1.8) admits a unique $C^{1}$ solution $u=u(t, x)$ on the domain $R(T)=\{(t, x) \mid 0 \leq t \leq T, 0 \leq x \leq L\}$, which satisfies exactly the final condition (2.1). If we can do so, then the exact boundary controllability is realized.

Since the hyperbolic wave has a finite speed of propagation, in order to realize the exact boundary controllability, $T>0$ should be suitably large. It is the reason to consider the semi-global $C^{1}$ solution for our mixed problem.

When all the boundary functions $H_{i}(t)(i=1, \ldots, n)$ acting on both ends $x=0$ and $x=L$ are used to realize the exact boundary controllability, we get the two-sided exact boundary controllability, while, if, for instance, only the boundary functions $H_{r}(t)(r=1, \ldots, m)$ acting on one end $x=L$ are used to realize the exact boundary controllability, we get the one-sided exact boundary controllability.

For fixing the idea, in what follows we consider only the one-sided exact boundary controllability. In this case, we should suppose that

$$
T>L\left(\max _{r=1, \ldots, m} \frac{1}{\left|\lambda_{r}(0)\right|}+\max _{s=m+1, \ldots, n} \frac{1}{\lambda_{s}(0)}\right) .
$$

The two-sided exact boundary controllability can be similarly discussed [16, 19-20].

The whole framework of resolution is to use a simple constructive method to construct a $C^{1}$ solution $u=u(t, x)$ to system (1.1) on the domain $R(T)=\{(t, x) \mid$ $0 \leq t \leq T, 0 \leq x \leq L\}$, such that it satisfies simultaneously the initial condition (1.6), the final condition (2.1) and the boundary condition (1.7) on the end $x=0$ without control. Once such a $C^{1}$ solution $u=u(t, x)$ is obtained, substituting it into the boundary condition (1.8), we finally get the boundary controls $H_{r}(t)(r=1, \ldots, m)$ on the end $x=L$, and then the desired one-sided exact boundary controllability.

To find such a $C^{1}$ solution $u=u(t, x)$ on the domain $R(T)$ is a non-standard problem. There is no uniqueness for this problem, however, the solution to this problem can be obtained by solving some well-posed mixed initial-boundary value problems, then the whole procedure of resolution possesses the stability.

Noting (2.2), there exists $\varepsilon_{0}>0$ so small that

$$
T>L\left(\sup _{|u| \leq \varepsilon_{0}} \max _{r=1, \ldots, m} \frac{1}{\left|\lambda_{r}(u)\right|}+\sup _{|u| \leq \varepsilon_{0}} \max _{s=m+1, \ldots, n} \frac{1}{\lambda_{s}(u)}\right) .
$$

Let

$$
T_{1}=L \sup _{|u| \leq \varepsilon_{0}} \max _{r=1, \ldots, m} \frac{1}{\left|\lambda_{r}(u)\right|}
$$

and

$$
T_{2}=L \sup _{|u| \leq \varepsilon_{0}} \max _{s=m+1, \ldots, n} \frac{1}{\lambda_{s}(u)} .
$$

Obviously, $T>T_{1}+T_{2}$.

The constructive method can be divided into several steps. 
(i) We first consider the forward mixed initial-boundary value problem for system (1.1) with the initial condition (1.6), the boundary condition (1.7) on $x=0$ and the following artificial boundary condition

$$
x=L: \quad v_{r}=f_{r}(t) \quad(r=1, \ldots, m),
$$

where $f_{r}(t)(r=1, \ldots, m)$ are any given functions of $t$ with small $C^{1}\left[0, T_{1}\right]$ norm, such that the conditions of $C^{1}$ compatibility are satisfied at the point $(t, x)=(0, L)$. By Lemma 1.1, there exists a unique semi-global $C^{1}$ solution $u=u_{f}(t, x)$ with small $C^{1}$ norm on the domain $R_{f}=\left\{(t, x) \mid 0 \leq t \leq T_{1}, 0 \leq x \leq L\right\}$. In particular, we have

$$
\left|u_{f}(t, x)\right| \leq \varepsilon_{0}, \quad \forall(t, x) \in R_{f} .
$$

Thus we can determine the value of $u=u_{f}(t, x)$ at $x=0$ as

$$
x=0: u_{f}=a(t), \quad 0 \leq t \leq T_{1}
$$

and the $C^{1}\left[0, T_{1}\right]$ norm of $a(t)$ is suitably small (Figure 1 ).

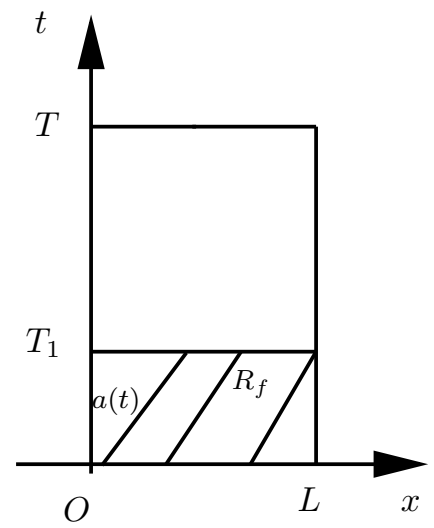

FIG. 1

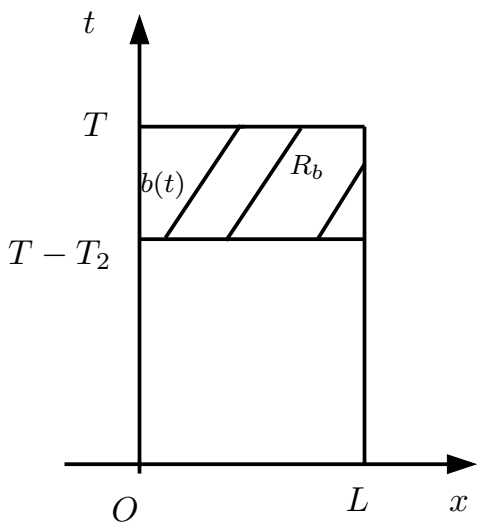

FIG. 2

(ii) In order to solve a corresponding backward mixed initial-boundary value problem, we assume that in a neighbourhood of $u=0$, the boundary condition (1.7) on the end $x=0$ without control can be equivalently rewritten as

$$
x=0: \quad v_{r}=\bar{G}_{r}\left(t, v_{m+1}, \ldots, v_{n}\right)+\bar{H}_{r}(t) \quad(r=1, \ldots, m)
$$

with

$$
\bar{G}_{r}(t, 0, \ldots, 0) \equiv 0 \quad(r=1, \ldots, m) .
$$

Similarly, we consider the backward mixed initial-boundary value problem for system (1.1) with the final condition (2.1), the boundary condition (2.9) (namely, (1.7)) on $x=0$, and the following artificial boundary condition

$$
x=L: \quad v_{s}=g_{s}(t) \quad(s=m+1, \ldots, n),
$$

where $g_{s}(t)(s=m+1, \ldots, n)$ are any given functions of $t$ with small $C^{1}\left[T-T_{2}, T\right]$ norm, satisfying the conditions of $C^{1}$ compatibility at the point $(t, x)=(T, L)$. By 
Lemma 1.1, there exists a unique semi-global $C^{1}$ solution $u=u_{b}(t, x)$ with small $C^{1}$ norm on the domain $R_{b}=\left\{(t, x) \mid T-T_{2} \leq t \leq T, 0 \leq x \leq L\right\}$. In particular, we have

$$
\left|u_{b}(t, x)\right| \leq \varepsilon_{0}, \quad \forall(t, x) \in R_{b} .
$$

Thus we can determine the value of $u=u_{b}(t, x)$ at $x=0$ as

$$
x=0: \quad u_{b}=b(t), \quad T-T_{2} \leq t \leq T
$$

and the $C^{1}\left[T-T_{2}, T\right]$ norm of $b(t)$ is suitably small (Figure 2).

(iii) Since both $a(t)$ and $b(t)$ satisfy the boundary condition (1.7) on $x=0$, noting $T>T_{1}+T_{2}$, we can find a $C^{1}[0, T]$ function $c(t)$ with small $C^{1}$ norm, such that

$$
c(t)= \begin{cases}a(t), & 0 \leq t \leq T_{1}, \\ b(t), & T-T_{2} \leq t \leq T\end{cases}
$$

and $c(t)$ satisfies the boundary condition (1.7) on $x=0$ for the whole interval $[0, T]$.

Now we change the role of the variables $t$ and $x$, then system (1.1) is equivalently rewritten as (1.19), namely,

$$
\frac{\partial u}{\partial x}+A^{-1}(u) \frac{\partial u}{\partial t}=\widetilde{B}(u) \stackrel{\text { def. }}{=} A^{-1}(u) B(u)
$$

with

$$
\widetilde{B}(0)=0 .
$$

We now consider the rightward mixed initial-boundary value problem (Figure 3) for system (1.19) (namely, (1.1)) with the initial condition

$$
x=0: \quad u=c(t), \quad 0 \leq t \leq T
$$

and the following boundary conditions reduced from the initial data $\varphi(x)$ and the final data $\Phi(x)$ :

$$
\begin{gathered}
t=0: v_{s}=l_{s}(\varphi(x)) \varphi(x) \quad(s=m+1, \ldots, n), \quad 0 \leq x \leq L, \\
t=T: v_{r}=l_{r}(\Phi(x)) \Phi(x) \quad(r=1, \ldots, m), \quad 0 \leq x \leq L .
\end{gathered}
$$

Still by Lemma 1.1, there exits a unique semi-global $C^{1}$ solution $u=u(t, x)$ with small $C^{1}$ norm on the domain $R(T)=\{(t, x) \mid 0 \leq t \leq T, 0 \leq x \leq L\}$. In particular, we have

$$
|u(t, x)| \leq \varepsilon_{0}, \quad \forall(t, x) \in R(T) .
$$




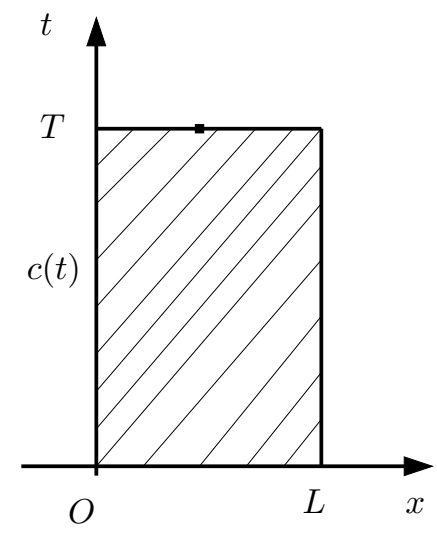

FIG. 3

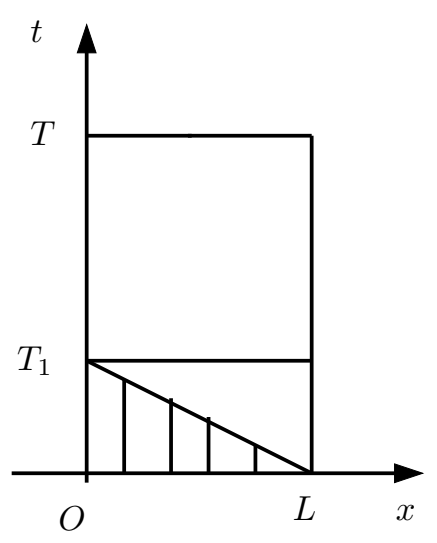

FIG. 4

(iv) In order to finish the construction, it is only necessary to check that $u=u(t, x)$ verifies the initial condition (1.6) and the final condition (2.1).

In fact, the $C^{1}$ solutions $u=u(t, x)$ and $u=u_{f}(t, x)$ satisfy the same system (1.19) (namely, (1.1)), the same initial condition

$$
x=0: \quad u=a(t), \quad 0 \leq t \leq T_{1}
$$

and the same boundary condition (2.16). By Lemma 1.3, i.e., the uniqueness of $C^{1}$ solution for this kind of one-sided mixed initial-boundary value problem, and noting the choice of $T_{1}$ given by (2.4), it is easy to see that on the domain

$$
\left\{(t, x) \mid 0 \leq t \leq \frac{T_{1}}{L}(L-x), 0 \leq x \leq L\right\}
$$

(Figure 4) we have

$$
u(t, x) \equiv u_{f}(t, x)
$$

In particular, we obtain (1.6). We can get (2.1) in a similar way.

Thus we realize the one-sided exact boundary controllability.

3. Exact boundary controllability of nodal profile. Recently, stimulated by some practical applications, Gugat et al. [10] proposed another kind of exact boundary controllability, called the nodal profile control. Different from the usual exact boundary controllability, this kind of controllability does not ask to exactly attain any given final state at a suitable time $t=T$ by means of boundary controls, instead it asks the state to exactly fit any given profile on one or some nodes after a suitable time $t=T$ by means of boundary controls. This kind of controllability which will be certainly applicable in many practical situations is called the exact boundary controllability of nodal profile in this paper.

More precisely, the exact boundary controllability of nodal profile on a boundary node $x=L$ can be defined as follows: For any given $C^{1}$ initial data $\varphi(x)$ and any given $C^{1}$ boundary functions $H_{r}(t)(r=1, \cdots, m)$, satisfying the conditions of $C^{1}$ compatibility at the point $(t, x)=(0, L)$, for any given $C^{1}$ vector function $\overline{\bar{u}}(t)$, if there exist $T>0$ and $C^{1}$ boundary controls $H_{s}(t)(s=m+1, \cdots, n)$ such that the $C^{1}$ solution $u=u(t, x)$ to the mixed initial-boundary value problem (1.1) and 
(1.6)-(1.8) fits exactly $\overline{\bar{u}}(t)$ on $x=L$ for $t \geq T$, then we have the exact boundary controllability of nodal profile on the boundary node $x=L$.

In this definition, when $t \geq T$, the value of solution $u=\overline{\bar{u}}(t)$ on $x=L$ should satisfy the boundary condition $(1.8)$, in which $v_{i}=\overline{\bar{v}}_{i}(t) \stackrel{\text { def. }}{=} l_{i}(\overline{\bar{u}}(t)) \overline{\bar{u}}(t)(i=1, \cdots, n)$. Hence, the requirement that the solution $u=u(t, x)$ fits exactly the given value $\overline{\bar{u}}(t)$ on $x=L$ for $t \geq T$ is equivalent to ask that $v_{s}(s=m+1, \cdots, n)$ fit exactly the given values $\overline{\bar{v}}_{s}(t)(s=m+1, \cdots, n)$ on $x=L$ for $t \geq T$, then the value of $v_{r}(r=1, \cdots, m)$ on $x=L$ for $t \geq T$ can be determined by the boundary condition (1.8) as follows:

$$
v_{r}=\overline{\bar{v}}_{r}(t) \stackrel{\text { def. }}{=} G_{r}\left(t, \overline{\bar{v}}_{m+1}(t), \cdots, \overline{\bar{v}}_{n}(t)\right)+h_{r}(t)(r=1, \cdots, m) .
$$

Let

$$
T>L \max _{s=m+1, \cdots, n} \frac{1}{\lambda_{s}(0)}
$$

and $\bar{T}$ be an arbitrarily given number such that

$$
\bar{T}>T \text {. }
$$

For any given initial data $\varphi(x)$ with small $C^{1}$ norm $\|\varphi\|_{C^{1}[0, L]}$ and any given boundary functions $H_{r}(t)(r=1, \cdots, m)$ with small $C^{1}$ norms $\left\|H_{r}\right\|_{C^{1}[0, \bar{T}]}(r=1, \cdots, m)$, satisfying the conditions of $C^{1}$ compatibility at the point $(t, x)=(0, L)$, suppose that the given values $\overline{\bar{v}}_{s}(t)(s=m+1, \cdots, n)$ on $x=L$ for $T \leq t \leq \bar{T}$ possess small $C^{1}$ norms $\left\|\overline{\bar{v}}_{s}\right\|_{C^{1}[T, \bar{T}]}(s=m+1, \cdots, n)$, then there exist boundary controls $H_{s}(t)(s=m+1, \cdots, n)$ with small $C^{1}$ norms $\left\|H_{s}\right\|_{C^{1}[0, \bar{T}]}(s=m+1, \cdots, n)$, such that the mixed initial-boundary value problem (1.1) and (1.6)-(1.8) admits a unique $C^{1}$ solution $u=u(t, x)$ with small $C^{1}$ norm on the domain $R(\tilde{T})=\{(t, x) \mid 0 \leq t \leq$ $\bar{T}, 0 \leq x \leq L\}$, which fits exactly the given values $v_{s}=\overline{\bar{v}}_{s}(t)(s=m+1, \cdots, n)$, namely, the given value $u=\overline{\bar{u}}(t)$, on the boundary node $x=L$ for $T \leq t \leq \bar{T}$.

In order to realize this exact boundary controllability of nodal profile, it suffices to construct a $C^{1}$ solution $u=u(t, x)$ to system (1.1) on the domain $R(\bar{T})=\{(t, x) \mid 0 \leq$ $t \leq \bar{T}, 0 \leq x \leq L\}$, such that it satisfies the initial condition (1.6), the boundary condition (1.8) on $x=L$ for $0 \leq t \leq \bar{T}$ and the given values $v_{s}=\bar{v}_{s}(t)(s=$ $m+1, \cdots, n)$ or $u=\overline{\bar{u}}(t)$ on $x=L$ for $T \leq t \leq \bar{T}$. In fact, substituting this solution into the boundary condition (1.7), we immediately get the boundary controls $H_{s}(t)(s=m+1, \cdots, n)$ on $x=0$ for $0 \leq t \leq \bar{T}$ and then the exact boundary controllability of nodal profile [17].

This $C^{1}$ solution $u=u(t, x)$ can be obtained through the following steps.

(i) Noting (3.2), there exists $\varepsilon_{0}>0$ so small that

$$
T_{1}<T
$$

where

$$
T_{1}=\max _{|u| \leq \varepsilon_{0}} \max _{s=m+1, \cdots, n} \frac{L}{\lambda_{s}(u)} .
$$

On the domain $R\left(T_{1}\right)=\left\{(t, x) \mid 0 \leq t \leq T_{1}, 0 \leq x \leq L\right\}$ we solve a forward mixed initial-boundary value problem for system (1.1) with the initial condition (1.6), the boundary condition (1.8) on $x=L$ and the following artificial boundary condition

$$
x=0: v_{s}=g_{s}(t) \quad(s=m+1, \cdots, n),
$$


where $g_{s}(t)(s=m+1, \cdots, n)$ are any given $C^{1}$ functions with small $C^{1}\left[0, T_{1}\right]$ norm, satisfying the conditions of $C^{1}$ compatibility at the point $(t, x)=(0,0)$.

By Lemma 1.1, this forward mixed problem admits a unique semi-global $C^{1}$ solution $u=u_{f}(t, x)$ with small $C^{1}$ norm on $R\left(T_{1}\right)$ (Figure 5). In particular,

$$
\left|u_{f}(t, x)\right| \leq \varepsilon_{0}, \quad \forall(t, x) \in R\left(T_{1}\right) .
$$

Thus, we can determine the value $\bar{u}(t)\left(0 \leq t \leq T_{1}\right)$ of $u=u_{f}(t, x)$ on $x=L$ and its $C^{1}\left[0, T_{1}\right]$ norm is small.

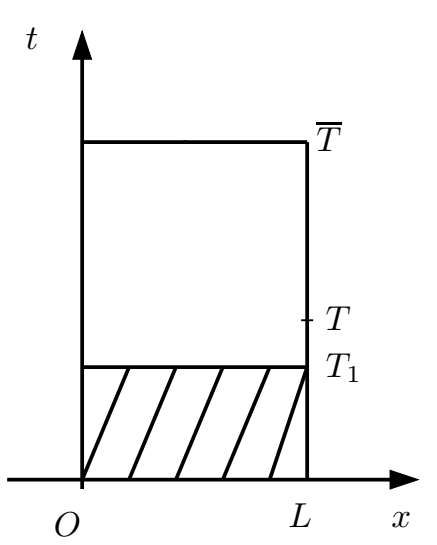

FiG. 5

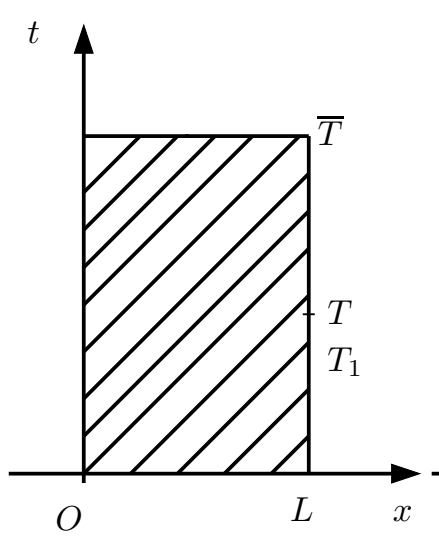

FiG. 6

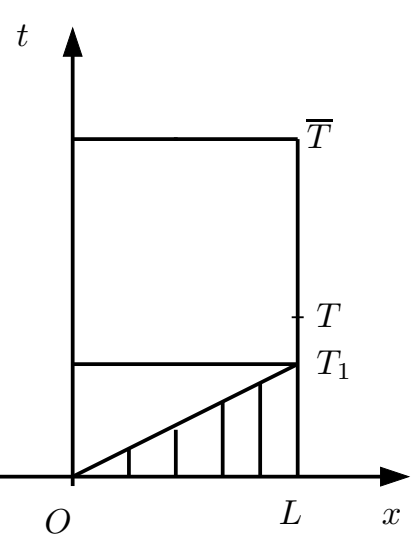

FIG. 7

Noting (3.4), there exists $u=u(t)$ on the interval $0 \leq t \leq \bar{T}$ with small $C^{1}[0, \bar{T}]$ norm, such that

$$
u(t)= \begin{cases}\bar{u}(t), & 0 \leq t \leq T_{1}, \\ \bar{u}(t), & T \leq t \leq \bar{T},\end{cases}
$$

and on the whole interval $0 \leq t \leq \bar{T}$ it satisfies the boundary condition (1.8), in which $v_{i}=v_{i}(t) \stackrel{\text { def. }}{=} l_{i}(u(t)) u(t)(i=1, \cdots, n)$.

(ii) We change the role of $t$ and $x$, and solve a leftward mixed initial-boundary value problem on $R(\bar{T})$ for system (1.1) with the initial condition

$$
x=L: u=u(t), \quad 0 \leq t \leq \bar{T},
$$

the boundary condition reduced from the original initial condition (1.6)

$$
t=0: v_{r}=v_{r}(x) \stackrel{\text { def. }}{=} l_{r}(\varphi(x)) \varphi(x) \quad(r=1, \cdots, m), 0 \leq x \leq L
$$

and the following artificial boundary condition

$$
t=\bar{T}: v_{s}=v_{s}(x) \quad(s=m+1, \cdots, n), 0 \leq x \leq L,
$$

where $v_{s}(x)(s=m+1, \cdots, n)$ are any given $C^{1}$ functions with small $C^{1}[0, L]$ norm and satisfy the conditions of $C^{1}$ compatibility at the point $(t, x)=(\bar{T}, L)$.

By Lemma 1.1, this leftward mixed problem admits a unique $C^{1}$ solution $u=$ $u(t, x)$ with small $C^{1}$ norm on the domain $R(\bar{T})$ (Figure 6). In particular,

$$
|u(t, x)| \leq \varepsilon_{0}, \quad \forall(t, x) \in R(\bar{T}) .
$$


(iii) This $C^{1}$ solution $u=u(t, x)$ satisfies system (1.1) and the boundary condition (1.8) on $x=L$. We now prove that $u=u(t, x)$ satisfies also the initial condition (1.6) at $t=0$.

For this purpose, consider the following one-sided mixed initial-boundary value problem for system (1.1) with the initial condition

$$
x=L: \quad u=\bar{u}(t), \quad 0 \leq t \leq T_{1}
$$

and the boundary condition (3.10). Both $u=u(t, x)$ and $u=u_{f}(t, x)$ are $C^{1}$ solutions to this one-sided mixed problem. Noting (3.5), (3.7) and (3.12), it is easy to see that the interval $0 \leq x \leq L$ on the initial axis $t=0$ is included in the maximum determinate domain of this one-sided mixed problem (Figure 7), hence, by Lemma 1.3, i.e., the uniqueness of $C^{1}$ solution to the one-sided mixed problem, $u=u(t, x)$ coincides with $u=u_{f}(t, x)$ on this interval $\{t=0,0 \leq x \leq L\}$, then $u=u(t, x)$ satisfies the initial condition (1.6) at $t=0$.

Thus, we get the desired exact boundary controllability of nodal profile.

4. Exact boundary observability. We still consider the forward mixed initialboundary value problem (1.1) and (1.6)-(1.8) under the hypotheses (1.3)-(1.4), (1.9)(1.10) and that the conditions of $C^{1}$ compatibility are satisfied at the points $(t, x)=$ $(0,0)$ and $(0, L)$, respectively.

For any preassigned $T>0$, if $\|\varphi\|_{C^{1}[0, L]}$ and $\|H\|_{C^{1}[0, T]}$ are small enough, then, by Lemma 1.1, this forward mixed problem admits a unique semi-global $C^{1}$ solution $u=u(t, x)$ with small $C^{1}$ norm on the domain $R(T)=\{(t, x) \mid 0 \leq t \leq T, 0 \leq x \leq L\}$.

The exact boundary observability asks to find a suitably large $T>0$ and certain boundary observed values on the interval $[0, T]$, such that the initial data $\varphi(x)$, then the whole $C^{1}$ solution $u=u(t, x)$ on the domain $R(T)$, can be uniquely determined by these boundary observed values on $[0, T]$. When boundary observed values on both ends $x=0$ and $x=L$ are used, we get the two-sided exact boundary observability, while, when boundary observed values only on one end, for instance, only on the end $x=0$, are used, we get the one-sided exact boundary observability.

In what follows, for fixing the idea, we consider only the one-sided exact boundary observability with the observation taken on the end $x=0$. The two-sided exact boundary observability can be similarly treated $[12,14,16]$.

For getting the exact boundary observability, the essential principle of choosing observed values on the boundary is that the observed values together with the boundary conditions can uniquely determine the value $u=\left(u_{1}, \ldots, u_{n}\right)$ on the boundary.

Following this principle, the observed value at $x=0$ should be essentially the diagonalized variables $v_{r}=\bar{v}_{r}(t)(r=1, \ldots, m)$ corresponding to the departing characteristics, then by means of the boundary condition (1.7), we get

$$
v_{s}=\bar{v}_{s}(t) \stackrel{\text { def. }}{=} G_{s}\left(t, \bar{v}_{1}(t), \ldots, \bar{v}_{m}(t)\right)+H_{s}(t) \quad(s=m+1, \ldots, n)
$$

and then, noting (1.9), we have

$$
\left\|\bar{v}_{s}\right\|_{C^{1}[0, T]} \leq C\left(\sum_{r=1}^{m}\left\|\bar{v}_{r}\right\|_{C^{1}[0, T]}+\left\|H_{s}\right\|_{C^{1}[0, T]}\right) \quad(s=m+1, \ldots, n) .
$$

Hence, the value of solution $u=\bar{u}(t)$ on $x=0$ satisfies

$$
\|\bar{u}\|_{C^{1}[0, T]} \leq C\left(\sum_{r=1}^{m}\left\|\bar{v}_{r}\right\|_{C^{1}[0, T]}+\sum_{s=m+1}^{n}\left\|H_{s}\right\|_{C^{1}[0, T]}\right) .
$$


Here and hereafter $C$ denotes a positive constant.

Let

$$
T>L\left(\max _{r=1, \ldots, m} \frac{1}{\left|\lambda_{r}(0)\right|}+\max _{s=m+1, \ldots, n} \frac{1}{\lambda_{s}(0)}\right)
$$

This requirement coincides with (2.2), the requirement for the one-sided exact boundary controllability.

We will prove that the boundary observed values $v_{r}=\bar{v}_{r}(t)(r=1, \ldots, m)$ at $x=0$ on the interval $[0, T]$ can be used to uniquely determine the initial data $\varphi(x)$ and we have the following observability inequality

$$
\|\varphi\|_{C^{1}[0, L]} \leq C\left(\sum_{r=1}^{m}\left\|\bar{v}_{r}\right\|_{C^{1}[0, T]}+\|H\|_{C^{1}[0, T]}\right) .
$$

By the constructive method, the whole proof is divided into the following steps.

(i) We change the role of $t$ and $x$ and consider the rightward Cauchy problem for system (1.1) with the initial condition

$$
x=0: \quad u=\bar{u}(t), \quad 0 \leq t \leq T
$$

determined uniquely by the observed values $v_{r}=\bar{v}_{r}(t)(r=1, \ldots, m)$ at $x=0$ and the boundary condition (1.7).

By Lemma 1.2, this Cauchy problem admits a unique global $C^{1}$ solution $u=$ $\tilde{u}(t, x)$ on the whole maximum determinate domain and

$$
\|\tilde{u}\|_{C^{1}} \leq C\left(\sum_{r=1}^{m}\left\|\bar{v}_{r}\right\|_{C^{1}[0, T]}+\sum_{s=m+1}^{n}\left\|H_{s}\right\|_{C^{1}[0, T]}\right) .
$$

We suppose that the observed values are accurate, i.e., there is no measuring error in the observation. Thus, $u=\tilde{u}(t, x)$ is the restriction of the solution $u=u(t, x)$ to the original mixed problem on the corresponding domain.

Noting (4.4) and the smallness of the data, this maximum determinate domain must intersect $x=L$ (Figure 8). Then, there exists $T_{0}\left(0<T_{0}<T\right)$ such that the value $\hat{u}(x)$ of the solution $u=u(t, x)$ on $t=T_{0}$ can be uniquely determined by $u=\tilde{u}(t, x)$ and

$$
\|\hat{u}\|_{C^{1}[0, L]} \leq C\left(\sum_{r=1}^{m}\left\|\bar{v}_{r}\right\|_{C^{1}[0, T]}+\sum_{s=m+1}^{n}\left\|H_{s}\right\|_{C^{1}[0, T]}\right) .
$$




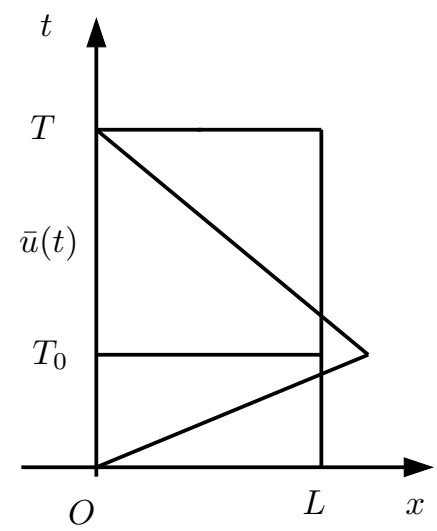

FIG. 8

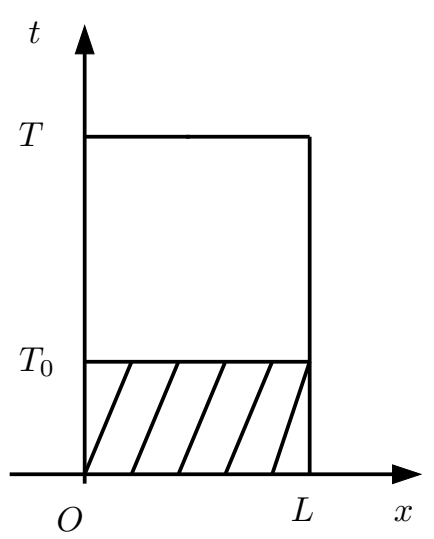

FIG. 9

(ii) We now solve the backward mixed initial-boundary value problem (Figure 9) for system (1.1) with the final condition

$$
t=T_{0}: \quad u=\hat{u}(x), \quad 0 \leq x \leq L,
$$

the boundary condition given by the observed values

$$
x=0: \quad v_{r}=\bar{v}_{r}(t) \quad(r=1, \ldots, m)
$$

and the boundary condition (1.8) on $x=L$, which, corresponding to the backward problem, should be supposed to be equivalently rewritten in a neighbourhood of $u=0$ as

$$
x=L: \quad v_{s}=\bar{G}_{s}\left(t, v_{1}, \ldots, v_{m}\right)+\bar{H}_{s}(t) \quad(s=m+1, \ldots, n)
$$

with

$$
\bar{G}_{s}(t, 0, \ldots, 0) \equiv 0 \quad(s=m+1, \ldots, n),
$$

then

$$
\sum_{s=m+1}^{n}\left\|\bar{H}_{s}\right\|_{C^{1}[0, T]} \leq C \sum_{r=1}^{m}\left\|H_{r}\right\|_{C^{1}[0, T]} .
$$

By Lemma 1.1, $u=u(t, x)$ as the $C^{1}$ solution to this mixed problem on the domain $R\left(T_{0}\right)=\left\{(t, x) \mid 0 \leq t \leq T_{0}, 0 \leq x \leq L\right\}$ satisfies

$$
\|u\|_{C^{1}\left[R\left(T_{0}\right)\right]} \leq C\left(\sum_{r=1}^{m}\left\|\bar{v}_{r}\right\|_{C^{1}[0, T]}+\|H\|_{C^{1}[0, T]}\right) .
$$

In particular, we get the desired observability inequality (4.5).

Thus, we realize the one-sided exact boundary observability. In this constructive way, the observability inequality (4.5) as an inverse inequality becomes a direct consequence of several direct inequalities obtained by solving some well-posed problems.

REMARK 1. The estimates on the controllability time (2.2), on the observability time (4.4) and on the nodal controllability time (3.2) are all sharp (cf. [5-9]). 
REMARK 2. The constructive method presented in this paper can be modified and generalized to get the exact boundary controllability, the exact boundary controllability of nodal profile and the exact boundary observability on a tree-like network with general topology [5-9].

REMARK 3. It would be very useful and interesting to realize numerically the constructive method suggested in this paper.

\section{REFERENCES}

[1] A. Bressan and G. M. Coclite, On the boundary control of systems of conservation laws, SIAM. J. Control Optim., 41 (2002), pp. 607-622.

[2] M. Cirinà, Boundary controllability of nonlinear hyperbolic systems, SIAM J. Control Optim., 7 (1969), pp. 198-212.

[3] M. CiRInÀ, Nonlinear hyperbolic problems with solutions on preassigned sets, Michigan Math. J., 17 (1970), pp. 193-209.

[4] O. Yu. Emanullov, Boundary control by semilinear evolution equations, Russian Math. Surveys, 44:3 (1989), pp. 183-184.

[5] QILOng Gu And TATsien Li, Exact boundary observability of unsteady flows in a tree-like network of open canals, Math. Meth. Appl. Sci., 32 (2009), pp. 395-418.

[6] Qilong Gu and TAtsien Li, Exact boundary controllability for quasilinear hyperbolic systems on a tree-like network and its applications, to appear.

[7] QILONG Gu AND TATsien Li, Exact boundary controllability for quasilinear wave equations in a planar tree-like network of strings, Ann. I. H. Poincaré-AN, 26 (2009), pp. 2373-2384.

[8] QILONG Gu AND TATSIEN Li, Exact boundary observability for quasilinear wave equations in a planar tree-like nework of strings, J. Math. Pure et Appl., DOI: 10.1016/j.matpur.2010.06.001.

[9] QILONG Gu AND TATSIEn LI, Exact boundary controllability of nodal profile for quasilinear hyperbolic systems on a tree-like network, Math. Meth. Appl. Sci., DOI: 10.1002/mma 1410, 2010.

[10] M. Gugat, M. Herty and V. Schleper, Flow control in gas networks: exact controllability to a given demand, Math. Meth. Appl. Sci., DOI: 10.1002/mma 1394, 2010.

[11] I. LASIECKA AND R. TRIGGIANI, Exact controllability of semilinear abstract systems with applications to waves and plates boundary control problems, Appl. Math. Optim., 23 (1991), pp. 109-154.

[12] Tatsien Li, Observabilité exacte frontière pour des systèmes hyperboliques quasi-linéaires, C. R. Acad. Sci. Paris, Ser. I, 342 (2006), pp. 937-942.

[13] Tatsien Li, Exact boundary observability for 1-D quasilinear wave equations, Math. Meth. Appl. Sci., 29 (2006), pp. 1543-1553.

[14] TAtsien Li, Exact boundary observability for quasilinear hyperbolic systems, ESAIM: Control, Optimization and Calculus of Variations, 14 (2008), pp. 759-766.

[15] TAtsien Li, Controllability and observability: From ODEs to quasilinear hyperbolic systems, 6th International Congress on Industrial and Applied Mathematics (ICIAM07), Zürich, Switzerland, 16-20 July 2007, Invited Lectures (Editors: Rolf Jeltsch, Gerhard Wanner), European Mathematical Society, 2009, pp. 251-278.

[16] Tatsien Li, Controllability and Observability for Quasilinear Hyperbolic Systems, AIMS Series on Applied Mathematics, Vol. 3, American Institute of Mathematical Sciences \& Higher Education Press, Springfield, Beijing, 2010.

[17] Tatsien Li, Exact boundary controllability of nodal profile for quasilinear hyperbolic systems, Math. Meth. Appl. Sci., 33 (2010), pp. 2101-2106.

[18] Tatsien Li And Yi Jin, Semi-global $C^{1}$ solution to the mixed initial-boundary value problem for quasilinear hyperbolic systems, Chin. Ann. Math., 22B (2001), pp. 325-336.

[19] Tatsien Li And Bopeng RaO, Local exact boundary controllability for a class of quasilinear hyperbolic systems, Chin. Ann. Math., 23B (2002), pp. 209-218.

[20] TAtsien Li And Bopeng RAO, Exact boundary controllability for quasilinear hyperbolic systems, SIAM J. Control Optim., 41 (2003), pp. 1748-1755.

[21] Tatsien Li And Bopeng RaO, Strong (weak) exact controllability and strong (weak) exact observability for quasilinear hyperbolic systems, Chin. Ann. Math., 31B (2010), pp. 723742 . 
[22] Tatsien Li And Lixin Yu, Contrôlabilité exacte frontière pour les équations des ondes quasi linéaires unidimensionnelles, C. R. Acad. Sci. Paris, Série I, 337 (2003), pp. 271-276.

[23] Tatsien Li And Lixin Yu, Exact boundary controllability for 1-D quasilinear wave equations, SIAM J. Control Optim., 45 (2006), pp. 1074-1083.

[24] Tatsien Li and Wenci Yu, Boundary Value Problems for Quasillinear Hyperbolic Systems, Duke University Mahtematics Series V, 1985.

[25] J.-L. Lions, Contrôlabilité Exacte, Perturbations et Stabilisation de Systèmes Distribués, Vol. I, Masson, 1988.

[26] J.-L. Lions, Exact controllability, stabilization and perturbations for distributed systems, SIAM Rev., 30 (1988), pp. 1-68.

[27] D. L. Russell, Controllability and stabilizability theory for linear partial differential equations, Recent progress and open questions, SIAM Rev., 20 (1978), pp. 639-739.

[28] Lixin YU, Exact boundary controllability for higher order quasilinear hyperbolic equations, Appl. Math. J. Chinese Univ., 20B (2005), pp. 127-141.

[29] E. ZuAZuA, Exact controllability for the semilinear wave equation, J. Math. Pures et Appl., 69 (1990), pp. 1-31.

[30] E. ZuAZuA, Exact controllability for semilinear wave equations, Ann. Inst. H. Poincaré, Anal. Non Linéaire, 10 (1993), pp. 109-129. 
T. LI 